\title{
Revision Chiari Surgery in Young Children: Predictors and Outcomes
}

\author{
John K. Chae Jeffrey P. Greenfield \\ Department of Neurological Surgery, Weill Cornell Medical College/NewYork-Presbyterian Hospital, \\ New York, NY, USA
}

\section{Keywords \\ Chiari malformation · Revision surgery · Retroflexed odontoid · Craniocervical instability - Dysautonomia}

\begin{abstract}
Introduction: Children aged 0-6 years with Chiari malformation (CM) often present with atypical symptoms and require revision surgery more often than older children. We studied characteristics and outcomes of CM patients in this agegroup who underwent one or more revision surgeries to assess how often revision surgery is necessary and successful in this age-group. Methods: We retrospectively reviewed patients who were diagnosed with CM 1 or CM 1.5 and surgically treated with posterior fossa decompression (PFD) with or without duraplasty before their 7th birthday. Basic demographics, preoperative presentation, operative details, and postoperative outcomes were analyzed. Results: Forty patients (mean age $3.2 \pm 1.7$ years, $35 \%$ female) were reviewed. The most common presenting symptoms were headache, dysphagia, and respiratory problems. Eight patients required one or more revision surgeries $11.6 \pm 7.6$ months on average after their initial surgery. Comparing the revision and no revision groups, dysautonomia was significantly more common prior to initial surgery in the children requir-
\end{abstract}

ing revision ( 37.5 vs. $3.1 \%, p=0.02)$. The revision group also trended toward more dysphagia ( 75.0 vs. $46.9 \%, p=0.24$ ) and respiratory problems ( 75.0 vs. $40.6 \%, p=0.12$ ). The most frequent reasons for reoperation were symptom recurrence (6/8), residual posterior fossa compression (3/8), significant scar tissue formation (2/8), ventral brainstem compression $(1 / 8)$, and suspected craniocervical instability (1/8). Of the 8 children undergoing reoperation, surgery achieved symptom resolution or improvement in 5, while 3 had unchanged symptoms. Two patients underwent a third surgery, after which 1 showed improvement and the other did not. This last patient showed short-term improvement after a fourth surgery but had symptom recurrence 12 months later. Discussion/Conclusion: Oropharyngeal and respiratory problems are particularly common in children aged 0-6 years with CM. Presentation with dysautonomia or other signs of brainstem compression will often predict an additional surgery will be needed after an initial PFD. Symptom recurrence is the most frequent reason for reoperation, and revision surgeries lead to improved clinical outcomes in the majority but not all of these young patients. Surgery in very young children is successful, but reoperation should be integrated into an up-front discussion algorithm particularly in children with severe symptoms.

(c) 2021 The Author(s)

Published by S. Karger AG, Basel karger@karger.com www.karger.com/pne

Karger $\stackrel{\text { ' }}{5}$

BOPEN ACCESS
(C) 2021 The Author(s)

Published by S. Karger AG, Basel

This is an Open Access article licensed under the Creative Commons Attribution-NonCommercial-4.0 International License (CC BY-NC) (http://www.karger.com/Services/OpenAccessLicense), applicable to the online version of the article only. Usage and distribution for commercial purposes requires written permission.
Correspondence to:

Jeffrey P. Greenfield, jpgreenf@med.cornell.edu 


\section{Introduction}

Chiari malformation (CM) 1 is a hindbrain abnormality that is classically defined as herniation of the cerebellar tonsils by at least $3-5 \mathrm{~mm}$ below the foramen magnum [1]. While exertional occipital headaches are the most common symptom, CM 1 is associated with highly variable symptomatology, including neck and shoulder pain, vertigo, ataxia, visual disturbances, oropharyngeal problems, respiratory problems, sensory deficits, muscle weakness, incontinence, dysautonomia, and cognitive problems [2, 3]. CM 1.5 involves herniation of both the tonsils and brainstem, and CM 0.5 involves ventral brainstem compression often seen in young children $[4,5]$. The main surgical treatments for CM $0.5,1$, and 1.5 include some version of a posterior fossa decompression (PFD). In this report, we will use the term CM 1 broadly to include all radiographic variations of Chiari.

In the current literature, clinical characteristics and surgical outcomes for CM 1 have been relatively well explored in adults, adolescents, and school-aged children. However, in comparison, there have only been a very few studies on infants and toddlers with CM 1. Two separate case series on this age-group reported that in these young CM 1 patients, oropharyngeal and respiratory problems, such as dysphagia, snoring, and sleep apnea, were particularly common and that they generally had good surgical outcomes [6-8].

A widely recognized conundrum in surgical management of very young CM 1 patients is that a significant proportion of them experience symptom recurrence years after surgery and undergo multiple PFD surgeries. In 2 prior case series, approximately $20-30 \%$ received multiple surgeries for their CM 1 [6-8]. Given that these young children have distinct preoperative clinical characteristics, there is a need to better understand predictors of failed initial surgery and both the role and success profile for additional surgeries in this age-group. Our objectives were to analyze clinical characteristics of children aged 0-6 years who underwent multiple surgeries for their CM 1 , explore reasons for reoperation, and track their postoperative outcomes.

\section{Materials and Methods}

Approved by the Weill Cornell Medicine Institutional Review Board (\#19-07020505), we retrospectively reviewed 40 patients with radiographically diagnosed CM 1 or CM 1.5 who underwent surgical PFD with or without duraplasty during the first 7 years of life (i.e., aged 0-6 years) at New York Presbyterian Hospital-Wei-
11 Cornell between July 2012 and December 2019. All surgeries were performed by the senior author (J.P.G.). Our exclusion criteria included CM 2 and patients who received their first PFD at another institution. Basic demographics, associated conditions, preoperative symptoms, pre- and postoperative radiographic data, operative details, and postoperative outcomes were recorded.

We divided our cohort into those who received one or more revision surgeries (i.e., revision group) and those who did not (i.e., no revision group). For statistical analyses, we used $\mathrm{R}$ (version 4.0.0) with RStudio (version 1.2.5042) to conduct the Welch 2 -sample $t$ test for continuous variables and Fisher's exact test for count data for categorical variables. We defined statistical significance with an alpha level of 0.05 .

\section{Initial PFD}

Every patient in the cohort had initially undergone a PFD with or without duraplasty. The PFD itself consisted of suboccipital craniectomy and cervical (C1) laminectomy with the goal of widening the foramen magnum and relieving dorsal compression. Every duraplasty was completed with an autologous pericranial patch. In this age cohort, our default preference was bony decompression without duraplasty due to universally recognized concerns about posterior fossa dural venous lakes in young children. While there are no universally accepted selection criteria for offering or performing duraplasty, it was considered and added in cases with a significant compressive tonsillar herniation or syrinx if deemed safe.

\section{Results}

\section{Sample Characteristics}

We reviewed a total of 40 patients aged 0-6 years whose preoperative characteristics are summarized in Table 1 . The mean age of the cohort was $3.2 \pm 1.7$ years, and $35 \%$ of these patients were females. Overall, the 3 most common presenting symptoms were headaches (77.5\%), dysphagia (52.5\%), and respiratory problems (47.5\%). Other, less common symptoms included neck and shoulder pain (27.5\%), balance instability (37.5\%), paresthesia (27.5\%), and muscle weakness (17.5\%). Overall, $27.5 \%$ of our cohort had a radiographically detectable syrinx. No child experienced any postoperative complications, such as wound infection, pseudomeningocele, and cerebrospinal fluid (CSF) leak, after the initial surgery.

\section{Differences in Baseline Preoperative Characteristics between the Revision and No Revision Groups}

We next stratified our cohort into the revision and no revision groups to compare their preoperative baselines before their initial PFD surgeries (Table 1). The 2 groups did not significantly differ in sex (34.4\% female vs. $37.5 \%$ female, $p=1$ ), proportion of CM 1.5 (12.5 vs. $25.0 \%, p=$ 0.58 ), or presence of other associated conditions. The no revision group tended to be older at time of surgery ( 3.5 
Table 1. Preoperative baseline characteristics

\begin{tabular}{|c|c|c|c|c|}
\hline & $\begin{array}{l}\text { No revision } \\
(N=32)\end{array}$ & $\begin{array}{l}\text { Revision } \\
(N=8)\end{array}$ & $\begin{array}{l}\text { Total } \\
(N=40)\end{array}$ & $\begin{array}{l}p \\
\text { value }\end{array}$ \\
\hline Mean age (SD) & $3.5( \pm 1.6)$ & $2.3( \pm 1.8)$ & $3.2( \pm 1.7)$ & 0.10 \\
\hline \multicolumn{5}{|l|}{ Sex, $N(\%)$} \\
\hline Female & $11(34.4)$ & $3(37.5)$ & $14(35.0)$ & \multirow{2}{*}{1} \\
\hline Male & $21(65.6)$ & $5(62.5)$ & $26(65.0)$ & \\
\hline \multicolumn{5}{|l|}{ Radiographic Chiari type, $N(\%)$} \\
\hline 1 r & $28(87.5)$ & $6(75.0)$ & $34(85.0)$ & \multirow{2}{*}{0.58} \\
\hline 1.5 & $4(12.5)$ & $2(25.0)$ & $6(15.0)$ & \\
\hline \multicolumn{5}{|l|}{ Associated conditions, $N(\%)$} \\
\hline Syrinx & $11(34.4)$ & $0(0.0)$ & $11(27.5)$ & 0.08 \\
\hline Scoliosis & $5(15.6)$ & $0(0.0)$ & $5(12.5)$ & 0.56 \\
\hline Retroflexed odontoid & $1(3.1)$ & $2(25.0)$ & $3(7.5)$ & 0.10 \\
\hline Craniocervical instability & $0(0.0)$ & $0(0.0)$ & $0(0.0)$ & - \\
\hline Ehlers-Danlos syndrome & $4(12.5)$ & $0(0.0)$ & $4(10.0)$ & 0.57 \\
\hline Idiopathic intracranial hypertension & $0(0.0)$ & 1 (12.5) & $1(2.5)$ & 0.20 \\
\hline Tethered cord & $1(3.1)$ & $0(0.0)$ & $1(2.5)$ & 1 \\
\hline Craniosynostosis & $0(0.0)$ & $0(0.0)$ & $0(0.0)$ & - \\
\hline Chromosome abnormalities & $1(3.1)$ & $0(0.0)$ & $1(2.5)$ & 1 \\
\hline Congenital adrenal hyperplasia & $1(3.1)$ & $0(0.0)$ & $1(2.5)$ & 1 \\
\hline \multicolumn{5}{|l|}{ Initial surgery type, $N(\%)$} \\
\hline PFD & $13(40.6)$ & $5(62.5)$ & $18(45.0)$ & \multirow{2}{*}{0.43} \\
\hline PFDD & $19(59.4)$ & $3(37.5)$ & $22(55.0)$ & \\
\hline \multicolumn{5}{|l|}{ Preoperative symptoms, $N(\%)$} \\
\hline Headache & $23(71.9)$ & $8(100.0)$ & $31(77.5)$ & 0.16 \\
\hline Neck/shoulder pain & $8(25.0)$ & $3(37.5)$ & $11(27.5)$ & 0.66 \\
\hline Back pain & 7 (21.9) & $1(12.5)$ & $8(20.0)$ & 1 \\
\hline Extremity pain & $7(21.9)$ & $3(37.5)$ & $10(25.0)$ & 0.39 \\
\hline Dysphagia & $15(46.9)$ & $6(75.0)$ & $21(52.5)$ & 0.24 \\
\hline Respiratory problems & $13(40.6)$ & $6(75.0)$ & $19(47.5)$ & 0.12 \\
\hline Balance instability & $12(37.5)$ & $3(37.5)$ & $15(37.5)$ & 1 \\
\hline Dizziness/vertigo & $1(3.1)$ & $2(25.0)$ & $3(7.5)$ & 0.10 \\
\hline Muscle weakness & $6(18.8)$ & $1(12.5)$ & $7(17.5)$ & 1 \\
\hline Decreased hearing & $1(3.1)$ & $1(12.5)$ & $2(5.0)$ & 0.36 \\
\hline Tinnitus & $0(0.0)$ & $0(0.0)$ & $0(0.0)$ & - \\
\hline Paresthesia & $11(34.4)$ & $0(0.0)$ & $11(27.5)$ & 0.08 \\
\hline Visual symptoms & $7(21.9)$ & $1(12.5)$ & $8(20.0)$ & 1 \\
\hline Urinary dysfunction & $3(9.4)$ & $2(25.0)$ & $5(12.5)$ & 0.26 \\
\hline Loss of consciousness & $2(6.3)$ & $1(12.5)$ & $3(7.5)$ & 0.50 \\
\hline Cognitive problems & $11(34.4)$ & $1(12.5)$ & $12(30.0)$ & 0.40 \\
\hline Dysautonomia & $1(3.1)$ & $3(37.5)$ & $4(10.0)$ & 0.02 \\
\hline Functionality loss & $15(46.9)$ & $2(25.0)$ & $17(42.5)$ & 0.43 \\
\hline
\end{tabular}

PFD, posterior fossa decompression without duraplasty; PFDD, posterior fossa decompression with duraplasty; SD, standard deviation. \pm 1.6 years vs. $2.3 \pm 1.8$ years, $p=0.10$ ). Radiographically, the no revision group also trended more toward the presence of syrinx ( 34.4 vs. $0.0 \%, p=0.08$ ) and scoliosis ( 15.6 vs. $0.0 \%, p=0.56)$, while the revision group tended to present with a retroflexed odontoid (3.1 vs. $25.0 \%, p=$ $0.10)$. The average durations of follow-up were 13.5 (range 0.2-72.7) months for the no revision group, 24.9 (range 6.3-55.0) months for the revision group, and 15.8 (range 0.2-72.7) months for the entire cohort.
Dysautonomia is a complex disorder to define but typically involves multiple signs and symptoms, such as tachycardia, temperature regulation problems, and unexplained gastrointestinal dysmotility. Four patients in our cohort carried a formal diagnosis with evaluation from cardiology. The presence of dysautonomia as a specific symptom category significantly differed between the 2 groups. Overall, $37.5 \%$ of the revision group had dysautonomia prior to their initial surgery, compared to $3.1 \%$ of 
Fig. 1. Sequences of surgeries performed. Initially, 18 and 22 patients underwent PFD without duraplasty and with duraplasty, respectively. $5 / 18$ patients from the no duraplasty group and $3 / 22$ patients from the duraplasty group had a subsequent procedure. PFD, posterior fossa decompression without duraplasty; PFDD, posterior fossa decompression with duraplasty; OCF, occipito-cervical fusion.

Table 2. Reasons for reoperation and clinical outcomes following multiple surgeries

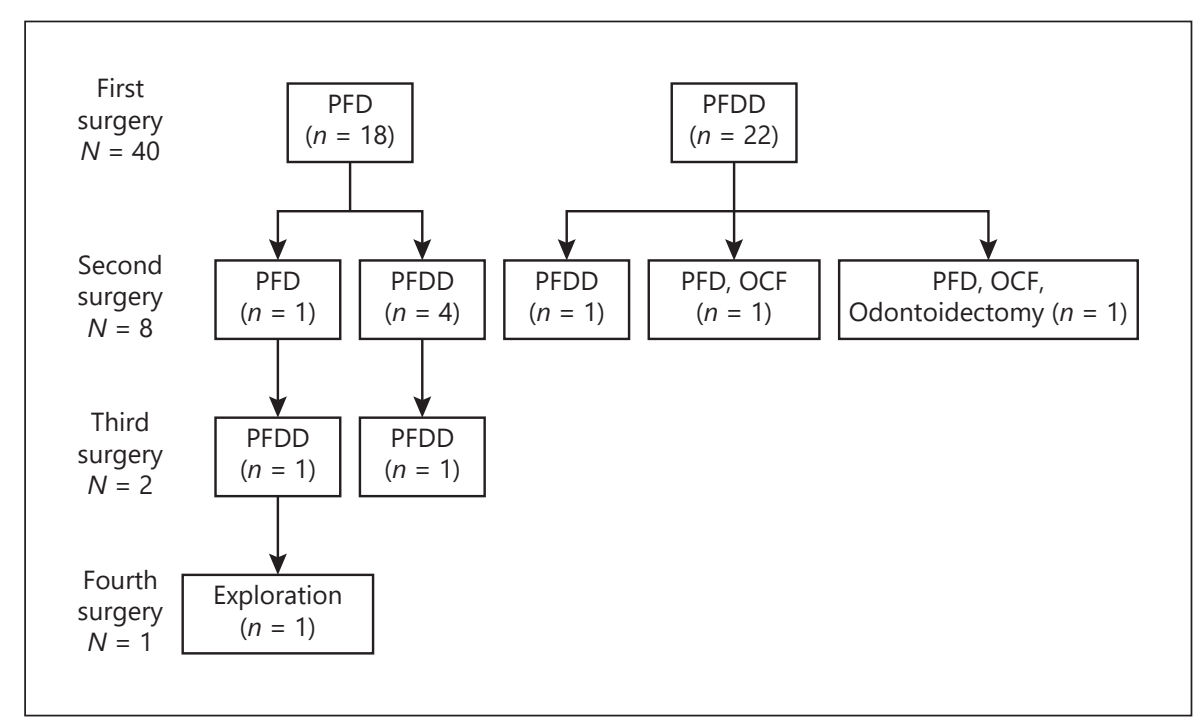

\begin{tabular}{llll}
\hline & $\begin{array}{l}\text { Surgery } 2 \\
(N=8)\end{array}$ & $\begin{array}{l}\text { Surgery } 3 \\
(N=2)\end{array}$ & $\begin{array}{l}\text { Surgery 4 } \\
(N=1)\end{array}$ \\
\hline $\begin{array}{l}\text { Reasons for reoperation } \\
\text { Symptom nonresolution }\end{array}$ & 1 & 0 & 0 \\
$\quad$ Symptom recurrence & 6 & 2 & 1 \\
$\quad$ New evidence of craniocervical instability & 1 & 0 & 0 \\
$\quad$ Progression of ventral compression & 1 & 0 & 0 \\
Suspicion of significant scar tissue & 2 & 0 & 0 \\
Residual dorsal compression & 3 & 1 & 0 \\
Mean time to reoperation since last surgery, months & $11.6 \pm 7.6$ & 9.3 & 26.1 \\
Clinical outcomes & 2 & 0 & 0 \\
$\quad$ Completely resolved & 2 & 1 & 0 \\
Improved & 3 & 1 & 1 \\
$\quad$ Unchanged & 0 & 0 & 0 \\
$\quad$ Worse & 0 & 0 & 0 \\
Postoperative complications & 8.2 & 24.7 & 13.3 \\
Mean follow-up period, months & & & \\
\hline
\end{tabular}

the no revision group $(p=0.02)$. Notably, the revision group also trended toward more headaches (71.9 vs. $100.0 \%, p=0.16$ ), dysphagia ( 46.9 vs. $75.0 \%, p=0.24$ ), and respiratory problems ( 40.6 vs. $75.0 \%, p=0.12)$. Back pain $(21.9$ vs. $12.5 \%, p=1)$ and paresthesias ( 34.4 vs. $0.0 \%, p=$ $0.08)$ were more common in the no revision group.

\section{Symptom Recurrence and Second Surgeries}

After the initial PFD surgeries, symptom recurrence rate was $6.3 \%$ in the no revision group and $75.0 \%$ in the revision group $(p<0.001)$. In the no revision group, these 2 patients with returning symptoms were, after discussion with families about medical versus surgical options, managed medically and did not require a revision sur- gery. In the revision group, such recrudescence of preoperative symptoms was the most common reason for a second surgery (Table 2). Other nonmutually exclusive reasons for reoperation included radiographic evidence of residual dorsal compression $(3 / 8,37.5 \%)$, significant scar tissue formation $(2 / 8,25.0 \%)$, progression of ventral brainstem compression secondary to a retroflexed odontoid $(1 / 8,12.5 \%)$, and suspected craniocervical instability $(1 / 8,12.5 \%)$. Of note, both cases with significant scar tissue formation on postoperative magnetic resonance imaging experienced substantial symptom progression.

Sequences of multiple surgeries performed are summarized in Figure 1. The mean time to reoperation since the first PFD was $11.6 \pm 7.6$ months. In the revision group, 

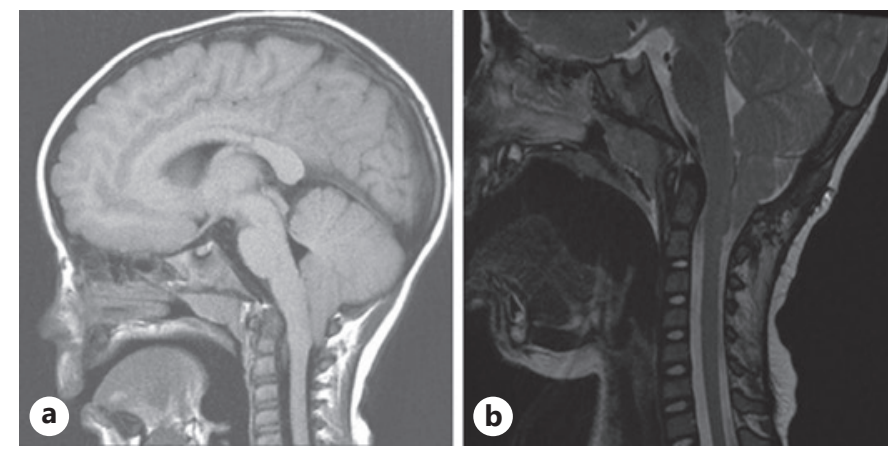

Fig. 2. A 2-year-old boy presented with exertional headaches, dysphagia, sleep apnea, speech difficulties, and jerking movements in the extremities. a Midsagittal T1-weighted MRI demonstrated CM 1.5 with a $15-\mathrm{mm}$ tonsillar herniation. He underwent a PFD with split-thickness duraplasty (i.e., splitting the outer dural layer), to which he initially showed a good clinical response. $\mathbf{b}$ His midsagittal T2-weighted MRI, 5 months after the surgery, showed improvement in the compression of the cervicomedullary junction.
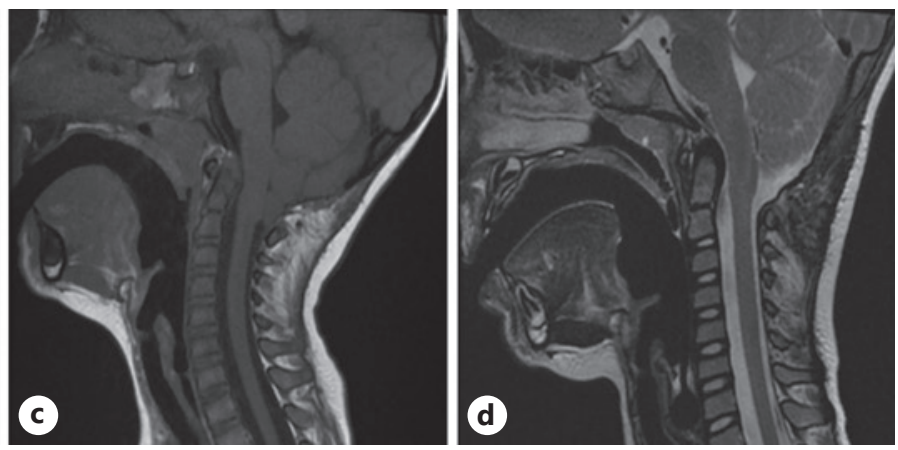

However, his symptoms recurred 12 months after the surgery. c Repeat MRI showed scar tissue formation and progressive dorsal compression. Twenty-one months after his initial PFD, he underwent bilateral tonsillopexy and an autologous duraplasty. d His 3-month postoperative MRI showed restoration of subarachnoid space, though he continued to experience intermittent symptoms MRI, magnetic resonance imaging; CM, Chiari malformation; $\mathrm{PFD}$, posterior fossa decompression.
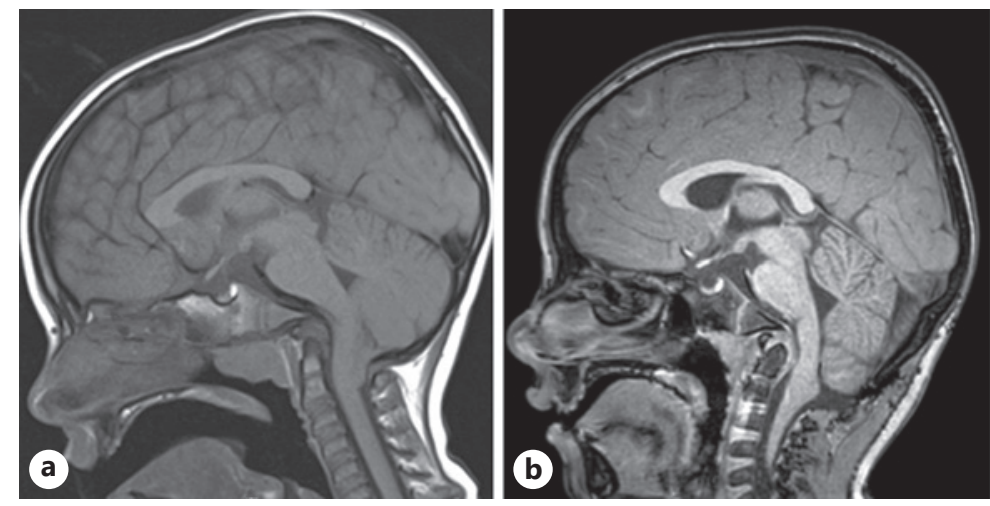

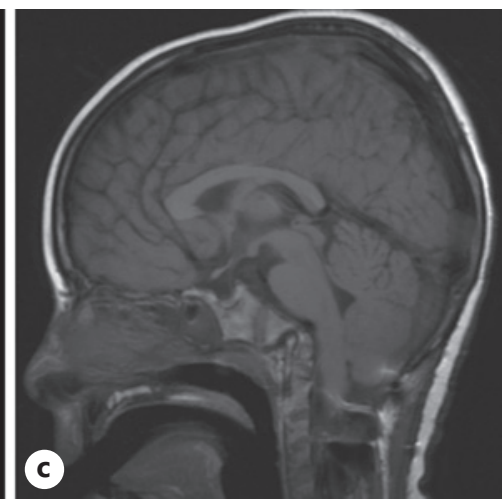

Fig. 3. A 4-year-old boy presented with exertional occipital headaches, neck pain, dysphagia, hoarseness, obstructive sleep apnea, dizziness, and visual disturbances. a Preoperative midsagittal T1weighted MRI showed CM 1.5 with a $16-\mathrm{mm}$ tonsillar herniation, platybasia, atlanto-occipital assimilation, and basilar invagination with a CXA of $121^{\circ}$. He received a PFD with bilateral tonsillopexy and autologous duraplasty knowing that his ventral compression might need to be subsequently addressed. Postoperatively, he had a few months of complete symptom resolution followed by recurrence of headaches, dysphagia, hoarseness, and sleep problems, which all improved on a C1 collar trial. b Midsagittal T1-weighted

5 patients had initially undergone an extradural PFD (i.e., without duraplasty) and 3 had undergone a PFD with duraplasty. The difference in reoperation rates between the 2 types of PFD was not significantly different (27.8\% PFD without duraplasty vs. $13.6 \%$ PFD with duraplasty, $p=$ 0.43 ), suggesting either approach carries the risk of requiring a second procedure in this age-group.

Revision Chiari Surgery in Young Children
MRI, 4 months after the initial PFD, showed adequate dorsal decompression but continued progression of ventral deformity with a CXA below $90^{\circ}$. Seven months after the first PFD, he underwent a multi-stage procedure involving a revision of suboccipital craniectomy, OCF (occiput to C4), and endoscopic endonasal odontoidectomy. c Midsagittal T1-weighted MRI, 11 months after the second surgery, demonstrated ventral decompression, and the patient continues to be symptom-free 3 years subsequent. CXA, clivo-axial angle; OCF, occipito-cervical fusion; MRI, magnetic resonance imaging; $\mathrm{CM}$, Chiari malformation; $\mathrm{PFD}$, posterior fossa decompression; C1, cervical.

In their second iteration of surgeries, 5 patients received a revision PFD with duraplasty (shown in Fig. 2) while only 1 got a repeat bone-only decompression. One patient with radiographic and symptomatological progression of previously identified retroflexed odontoid underwent a revision PFD with endonasal endoscopic odontoidectomy and occipito-cervical fusion (OCF) (shown in 

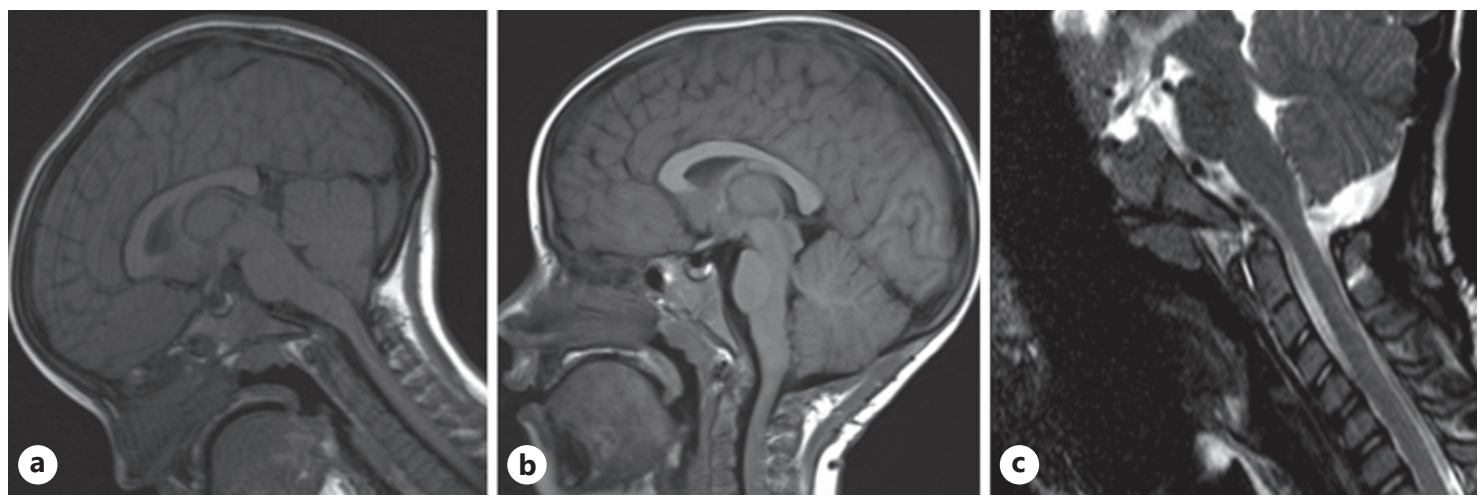

Fig. 4. A 5-year-old girl with a hereditary disease of connective tissue presented with exertional occipital headaches, dysphagia, GI dysmotility, and urinary incontinence. a Midsagittal T1-weighted MRI showed CM 1 with a 6-mm tonsillar herniation. She was managed with a PFD with an autologous duraplasty. b Postoperative MRI, 2 months later, showed restoration of subarachnoid space, but the symptoms were static. Despite normal flexion-extension C1 imaging, due to high clinical suspicion of hypermobility, she

Fig. 3). One patient who had high clinical suspicion for craniocervical instability, following a successful C1 collar trial with symptom amelioration, was treated with a revision PFD and OCF (shown in Fig. 4).

\section{Outcomes of Revision Surgeries}

After their second surgeries, 2 patients had complete symptom resolution, 3 showed significant clinical improvements, and 3 had relatively unchanged symptoms. No child experienced worsened symptoms (Table 2). There were no postoperative complications from the second surgery, including an absence of CSF leak, pseudomeningocele, or wound infection.

Among the 3 patients with unchanged long-term outcomes after their second surgeries, 2 underwent a third operation because of their significant recurrence and progression of baseline preoperative symptoms. After a repeat PFD with duraplasty, 1 patient reported long-term improvement while the other had short-term improvement with symptom recurrence 8 months later. This latter patient got a fourth surgery after which the patient reported 12 months of clinical improvement, followed by another substantial symptom recurrence. Notably, this patient was the only one in our cohort who had undergone the initial PFD before 12 months of age. Again, there were no postoperative complications from any of these additional surgeries. was placed in a C1 collar trial, which significantly improved all of her symptoms. Thus, she underwent a revision PFD with an OCF (occiput to C3). c Midsagittal T2-weighted MRI, 9 months after the second surgery, continued to display an adequate decompression, and the patient's symptoms remained greatly improved. MRI, magnetic resonance imaging; CM; Chiari malformation; PFD, posterior fossa decompression; OCF, occipito-cervical fusion; $\mathrm{C} 1$, cervical.

\section{Discussion}

There have only been a few case series reporting surgical management of infants and toddlers with CM 1. Perhaps this scarcity in the current literature is in part due to the relative infrequency with which the diagnosis is made in this young age-group. A study on a nationwide sample of CM 1 patients aged $0-18$ years derived from the US National Surgical Quality Improvement Program-Pediatric (NSQIP-P) database showed that the majority of patients treated surgically were aged 5-14 years [9].

This potential delay in diagnosis reflects challenges stemming from the complex nature of Chiari symptomatology. In the general CM 1 population, the onset of symptoms can be in the 2 nd or 3rd decade, but even with earlier onset, many patients do not seek medical attention until their early adulthood [1]. Though pediatric cases of CM 1 have increasingly been identified with the wider availability of magnetic resonance imaging, many young children present asymptomatically or atypically, and their naturally limited communication skills serve as a barrier to fully understanding their individual symptomatology $[8,10]$.

In our cohort of patients aged 0-6 years, headache was the most frequent preoperative symptom overall, followed by oropharyngeal and respiratory problems. Other "classic" Chiari symptoms, including neck and shoulder pain, sensory disturbances, balance instability, and visual symptoms were less common in this age-group. The rela- 
tive underreporting of these other symptoms could be due to fundamentally different underlying pathophysiology in this age-group and/or many children's inability to verbalize their symptoms. Regardless, the high prevalence of oropharyngeal and respiratory problems in our cohort was overall consistent with the findings in the prior case series [6-8]. Recognition of these symptom categories is, therefore, important for preventing a delay in diagnosis, which is associated with increased risk of headache and further neurologic symptoms [10].

\section{Predicting Which Patients Might Fail Their Initial PFD}

Considering that postoperative symptom recurrence and revision surgeries occur relatively frequently in the general CM 1 population, we were interested in better understanding this conundrum in our youngest cohort. We found that dysautonomia, a sign of ventral brainstem compression, was significantly more common in the revision group than in no revision group. We also noted that retroflexed odontoid, a known cause of ventral compression, tended to be more common in the revision group than in no revision group. Therefore, given that PFD surgeries predominantly improve dorsal compression, it makes sense that patients with progressive ventral pathophysiology would likely require additional surgeries after their initial PFD.

The 3 overall most common symptoms - headache, dysphagia, and respiratory problems - were also more prevalent in the revision group (100 vs. $71.9 \%$ for headache, 75.0 vs. $46.9 \%$ for dysphagia, and 75.0 vs. $40.6 \%$ for respiratory problems). These trends again highlight the potential significance of ventral brainstem compression, which can cause lower cranial nerve dysfunction [11]. It should be noted however that none of these differences in prevalence reached statistical significance most likely because we were underpowered.

Another notable preoperative trend was that all 11 children with a syrinx in our cohort were in the no revision group. Therefore, unsurprisingly, the no revision group trended toward more signs and symptoms attributable to syrinx, such as scoliosis, back pain, and paresthesia. However, it would be erroneous to conclude from these trends that children with syrinx are less likely to undergo reoperations. The more likely explanation is that these patients received a more aggressive PFD with dural opening and duraplasty, as per surgeon's routine practice to more definitively address their underlying syrinxes. Thus, it appears that the threshold for a repeat PFD was higher due to the more invasive initial procedure.

Revision Chiari Surgery in Young Children

\section{Making Decisions to Reoperate and Tracking Their}

\section{Outcomes}

Out of 40 patients, 8 received a second surgery. This $20 \%$ reoperation rate was consistent with those noted in the 2 prior case series on this age-group [6-8]. Our revision group consisted of 5 who had received PFD without duraplasty and 3 with duraplasty. In the literature, there is a considerable amount of conflicting data and controversy surrounding the risks and benefits of PFD with duraplasty. Consistent with our results, a recent systematic review and meta-analysis showed no significant difference in reoperation rate between the duraplasty and no duraplasty groups in pediatric cases [12].

However, at our institution, we often recommend a more conservative approach without duraplasty for very young children without syrinx because of their age, rapid rate of growth, and evidence in the literature that dural opening may be associated with more postoperative complications, despite our historically very low institutional complication rate with duraplasty in general $[13,14]$. We are more inclined to offer reoperation with duraplasty to young patients who have failed this extradural approach. This stepwise surgical algorithm likely explains why our duraplasty group trended toward, without statistical significance, fewer reoperations and why the revision group tended to be younger at time of initial surgery.

The majority of patients undergoing multiple surgeries experienced good clinical outcomes. The most common reason for reoperation was recurrence and subsequent progression of symptoms. In the general CM 1 population, symptom recurrence is a common source of frustration that can occur up to $35 \%$ of patients after surgery [15]. Among the 8 patients in our cohort who experienced symptom recurrence, 6 underwent a second surgery and the remaining 2 received medical management to address their symptoms.

When patients present with symptom recurrence after surgery, it is important to evaluate and look for their underlying pathophysiology. Some of our patients with symptom recrudescence showed significant scar tissue formation in the suboccipital region (as exemplified in Fig. 2c) on postoperative imaging. During their revision surgery, we invariably noticed that the previously resected posterior atlanto-occipital membrane overlying the dura had been scarred and caused restriction of the dura, requiring direct, sharp decompression [16]. Other patients showed residual dorsal compression on repeat postoperative imaging. This compression could be due to inadequate decompression from the initial surgery, ligamentous calcification, or frank bony regrowth. 
Some patients fail to respond fully to PFD because their underlying pathophysiology is related to indirect, rather than direct, compression within the posterior fossa. These etiologies may not be fully addressed by decompression surgery or may be unrelated to dorsal compression itself. These populations may include children with raised intracranial pressure or craniosynostosis. In our case series, 1 unusual child required an odontoidectomy with OCF for radiographically progressing retroflexed odontoid and worsening symptoms attributable to ventral brainstem compression. Craniocervical instability is another condition associated with complex CM 1 that could explain failure of PFD alone in some patients. We evaluated for clinically relevant craniocervical instability by assessing for any abnormal movement on flexion-extension imaging and placing the patient on a $\mathrm{C} 1$ collar trial to observe any symptom improvement. We had 1 patient who, despite showing a normal flexion-extension imaging, responded beautifully to a $\mathrm{C} 1$ collar trial. We suspected craniocervical instability in this unusual patient with the positive collar trial response and severe underlying connective tissue disorder, and we subsequently treated with an OCF. Both of these patients had very good long-term outcomes as their second surgeries directly addressed their underlying pathophysiology. However, it should be noted that we generally reserve OCF as a last resort in these young children because of their fragile and small osseous elements for instrumentation. Other potential causes of symptom persistence that are important to rule out include idiopathic intracranial hypertension, CSF leak, and tethered cord.

One unique child had 4 procedures beginning at 8 months of age with a very conservative decompression because of his age and focal compression at C1. Subsequent surgeries were performed to address residual dorsal compression from this early cautious approach. However, even though postoperative imaging after the third and fourth surgeries indicated adequate dorsal decompression, the patient continued to experience symptom recurrence, confounded by a late diagnosis of autism spectrum disorder. A point of diminishing returns after 2 decompressions is apparent, which would argue for either delaying surgery, if possible, until a more definitive decompression is feasible.

\section{Limitations and Future Directions}

There were limitations to this study. First, generalizability of our findings is limited by the single-centered, retrospective nature of the study with a relatively short mean postoperative follow-up period. Second, we had a small sample size for the revision group, so we were un- derpowered to detect smaller, more subtle statistical differences between the revision and no revision groups. Third, symptom tracking was particularly challenging in this age-group because many patients naturally lacked the necessary communication skills. Thus, we had to rely on their parents and/or behavioral signs to understand their symptomatology. Overall, a larger sample size is required to do more detailed studies on postoperative outcomes. Fourth, though our analysis showed that there was no significant difference in reoperation rates between those who initially received PFD without duraplasty and those with duraplasty and that the revision group contained both surgery types (5 without and 3 with duraplasty), there still could have been selection bias. To better understand the natural history of CM 1, it will be revealing to investigate the prevalence of asymptomatic infants with CM 1. Also, in this report, we have used the term CM 1 broadly to group different radiographic variations of Chiari, but our cohort did not contain any CM 0 or 0.5 , another potential area for further investigation.

\section{Conclusions}

Children aged 0-6 years with CM 1 present differently, with oropharyngeal and respiratory problems being particularly more common. Patients presenting with dysautonomia, a sign of ventral brainstem compression, may require additional surgeries after initial PFD. Symptom recurrence is a frequent reason for reoperation, and revision surgeries that address specific underlying pathophysiology lead to improved clinical outcomes in the majority of these young patients. Families of infants and toddlers should be counseled to the increased need for repeat procedures in these very young age-groups, outcomes of which tend to be favorable with careful preoperative patient selection focusing on pathophysiology and risk tolerance.

\section{Acknowledgment}

The authors would like to thank Ericka Deadwyler-Gourgue MS, AGPCNP-BC for coordinating care of Chiari patients.

\section{Statement of Ethics}

This study was approved by the Weill Cornell Medicine Institutional Review Board (\#19-07020505), which determined that written informed consent was not required for this study. 


\section{Conflict of Interest Statement}

Jeffrey P. Greenfield, MD, PhD is a member of the Editorial Board of Pediatric Neurosurgery. The authors otherwise have no conflicts of interest to declare.

\section{Funding Sources}

The authors have no funding sources to report.

\section{Author Contributions}

John Chae contributed to study design, data acquisition, data analysis, and manuscript preparation. Jeffrey Greenfield contributed to study design, data interpretation, and manuscript revision.

\section{Data Availability Statement}

The primary data used in this study are not publicly available for confidentiality reasons. Queries about the data should be directed to the corresponding author.

\section{References}

1 Milhorat TH, Chou MW, Trinidad EM, Kula RW, Mandell M, Wolpert C, et al. Chiari I malformation redefined: clinical and radiographic findings for 364 symptomatic patients. Neurosurgery. 1999;44(5):1005-17.

2 Massimi L, Frassanito P, Chieffo D, Tamburrini G, Caldarelli M. Bony decompression for chiari malformation type I: long-term followup. Acta Neurochir Suppl. 2019;125:119-24.

3 Steinberg SN, Greenfield JP, Perrine K. Neuroanatomic correlates for the neuropsychological manifestations of Chiari malformation type I. World Neurosurg. 2020 Apr;136:4629.

4 Tubbs RS, Iskandar BJ, Bartolucci AA, Oakes WJ. A critical analysis of the Chiari 1.5 malformation. J Neurosurg. 2004 Nov;101(2 Suppl):179-83.

5 Morgenstern PF, Tosi U, Uribe-Cardenas R, Greenfield JP. Ventrolateral tonsillar position defines novel Chiari 0.5 classification. World Neurosurg. 2020 Apr;136:444-53.

6 Greenlee JD, Donovan KA, Hasan DM, Menezes AH. Chiari I malformation in the very young child: the spectrum of presentations and experience in 31 children under age 6 years. Pediatrics. 2002 Dec;110(6):1212-9.
7 Albert GW, Menezes AH, Hansen DR, Greenlee JD, Weinstein SL. Chiari malformation Type I in children younger than age 6 years: presentation and surgical outcome. J Neurosurg Pediatr. 2010 Jun;5(6):554-61.

8 Grahovac G, Pundy T, Tomita T. Chiari type I malformation of infants and toddlers. Childs Nerv Syst. 2018 Jun;34(6):1169-76.

9 Vedantam A, Mayer RR, Staggers KA, Harris DA, Pan IW, Lam SK. Thirty-day outcomes for posterior fossa decompression in children with Chiari type 1 malformation from the US NSQIP-Pediatric database. Childs Nerv Syst. 2016 Nov;32(11):2165-71.

10 Aitken LA, Lindan CE, Sidney S, Gupta N, Barkovich AJ, Sorel M, et al. Chiari type I malformation in a pediatric population. Pediatr Neurol. 2009 Jun;40(6):449-54.

11 Grabb PA, Mapstone TB, Oakes WJ. Ventral brain stem compression in pediatric and young adult patients with Chiari I malformations. Neurosurgery. 1999 Mar;44(3):520-8; discussion 27-8.

12 Chai Z, Xue X, Fan H, Sun L, Cai H, Ma Y, et al. Efficacy of posterior fossa decompression with duraplasty for patients with chiari malformation type I: a systematic review and meta-analysis. World Neurosurg. 2018 May;113: 357-65.e1.
13 Hoffman CE, Souweidane MM. Cerebrospinal fluid-related complications with autologous duraplasty and arachnoid sparing in type I Chiari malformation. Neurosurgery. 2008 Mar;62(3 Suppl 1):156-1; discussion 60-1.

14 Shweikeh F, Sunjaya D, Nuno M, Drazin D, Adamo MA. National trends, complications, and hospital charges in pediatric patients with Chiari malformation type I treated with posterior fossa decompression with and without duraplasty. Pediatr Neurosurg. 2015;50(1): 31-7.

15 McGirt MJ, Attenello FJ, Atiba A, GarcesAmbrossi G, Datoo G, Weingart JD, et al. Symptom recurrence after suboccipital decompression for pediatric Chiari I malformation: analysis of 256 consecutive cases. Childs Nerv Syst. 2008 Nov;24(11):1333-9.

16 Tubbs RS, Wellons JC III, Oakes WJ, Blount JP. Reformation of the posterior atlanto-occipital membrane following posterior fossa decompression with subsequent constriction at the craniocervical junction. Pediatr Neurosurg. 2003 Apr;38(4):219-21. 